\title{
Estudio de los futuros comunicadores y su categoría de prosumidores en el contexto de la globalización
}

\section{Study of future communicators and their category of prosumers in the context of globalization}

\section{Estudo de futuros comunicadores e sua categoria de prosumers no contexto da globalização}

\author{
Julio Bravo Mancero ${ }^{1}$ \\ Universidad de Santiago de Compostela (España) \\ julio.bravo@rai.usc.es \\ Carlos Larrea Naranjo ${ }^{2}$ \\ Universidad de La Habana (Cuba) \\ carlos.larrea@fcom.uh.cu \\ Ramiro Ruales Parreño 3 \\ Universidad Nacional de Chimborazo (Ecuador) \\ rruales@unach.edu.ec
}

Fecha de recepción: 29 de febrero de 2019

Fecha de recepción evaluador: 25 de marzo de 2019

Fecha de recepción corrección: 29 de marzo de 2019

\footnotetext{
1 Julio Bravo Mancero es Doctor en Comunicación e Información Contemporánea de la Universidad Santiago de Compostela. https://orcid.org/0000-0002-4468-9247

${ }^{2}$ Carlos Larrea Naranjo es Doctor en Comunicación de la Universidad de La Habana; Magister Business Administration, Universidad Regional Autónoma de los Andes. https://orcid.org/0000-0003-4881-5666

${ }^{3}$ Ramiro Ruales Parreño es Doctor en Comunicación de la Universidad de La Habana; Magíster en Comunicación Corporativa por la Universidad Regional Autónoma de los Andes. https://orcid.org/0000$\underline{0003-4812-2085}$
} 


\title{
Resumen
}

El presente artículo reflexiona sobre el momento que vive, en la actualidad, la comunicación, por la presencia de los prosumidores. Este análisis pretende explicar, desde el basamento teórico, cómo en la práctica se produce los procesos de interacción y la interrelación. Es un estudio de tipo comunicacional, empleó técnicas de recolección de información cuali-cuantitativa; y definió como instrumentos: la encuesta, la entrevista y la observación. Los investigadores se plantearon como objetivo: Determinar cómo los futuros comunicadores asumen la categoría de prosumidores en el contexto de la globalización. Entre los resultados relevantes se demostró que, esencialmente son los jóvenes, quienes generan nuevos procesos de comunicación como pilar de la comunidad virtual.

Palabras clave: Prosumidores, prosumidores mediáticos, medios de comunicación, Tics, Web 2.0

\begin{abstract}
The present article reflects on the moment that the communication lives, at present, by the presence of the prosumers. This analysis aims to explain, from the theoretical base, how in practice the processes of interaction and inter-relationship are produced. It is a study of communicational type, used techniques of collection of quali-quantitative information; and defined as instruments: the survey, the interview and the observation. The researchers set themselves the objective: To determine how future communicators assume the category of prosumers in the context of globalization. Among the relevant results, it was shown that it is essentially young people who generate new communication processes as a pillar of the virtual community.
\end{abstract}

Keywords: prosumers, media prosumers, mass media, Tics, Web 2.0

\section{Resumo}

$\mathrm{O}$ artigo presente medita na atualidade no momento que vive, a comunicação, para a presença do prosumidores. Esta análise busca explicar, do porão teórico, como na prática ele/ela acontece o enterrar-ação processa e o enterrar-relação. É um estudo de comunicacional de tipo, usou técnico de ajuntamento de informação cuali-quantitativa; e definiu como instrumentos: a pesquisa, a entrevista e a observação. Os investigadores pensaram sobre como objetivo: Determinar como o comunicadores futuro assume a categoria de prosumidores no contexto do globalização. Entre os resultados excelentes foi demonstrado que, essencialmente elas são as mocidades que geram processos de comunicação novos como pilar da comunidade virtual.

Palavras teclam: Prosumidores, mediáticos de prosumidores, mídia, Tiques, Web 2.0 


\section{Introducción}

En todo proceso de cambio y adaptación a las formas de relación con los nuevos medios, pueden encontrarse tres acciones: componer, compartir/participar y difundir. De su análisis se ocupó el equipo de investigadores que desarrollaron este documento como parte del Observatorio de Medios y Estudios Sociales, de la carrera de Comunicación Social, de la Universidad Nacional de Chimborazo con el apoyo de los estudiantes de séptimo y octavo semestre de las cátedras Periodismo Digital 1 y 2.

La acción -componer- evidencia que, en una vídeo-denuncia, una llamada a la solidaridad mediante fotografías o noticias elaboradas por usuarios de internet, las posibilidades para crear mediante las nuevas tecnologías y dispositivos sobrepasan la imaginación de cualquiera; las generaciones anteriores contaban con medios y posibilidades limitadas. Ahora bien, los creadores de contenidos $-\mathrm{y}$ consumidores de los mismos- tienen un objetivo claro: compartirlos. Crear sin compartir no tiene sentido. Las redes sociales juegan un papel fundamental, ya que se constituyen en un medio en el que cualquier contenido tiene cabida. De hecho, surgen problemas precisamente porque los jóvenes no perciben los límites de la exhibición pública de los contenidos como quizás lo hagan los adultos.

El presente estudio, de tipo comunicacional, identificó los nuevos escenarios en los que se manejan los prosumidores motivados por la vorágine de la Era Digital que, abrió perspectivas diferentes que están conduciendo al cambio en la forma de interactuar en la vida cotidiana como una manera distinta de construir la realidad social.

Desde el análisis del prosumidor: compartir es participar. De hecho, se empieza a hablar de la cultura participativa de las audiencias; comenzando por el feed-back para criticar el tratamiento que se ha dado a un tema hasta ser testigo en primera persona de un hecho que se ha grabado; los medios digitales han permitido a los ciudadanos sentirse escuchados. Para fundamentar este manuscrito se utilizaron propuestas de autores, como: Albornoz (2011); Briggs (2007); Chomsky (1997); Friedman (2005); Habermas (1993); Herner y Tufte (2013); Levinson (1997); McLuhan (1996); Robertson (1992); Toffler (2006); Werner \& Weiss (2006).

La difusión, como elemento clave, para dar un peso específico a las posibilidades que los nuevos medios ofrecen al usuario como consumidor y como productor de contenidos, les brinda la oportunidad de llegar a millones de personas en un corto período, ventaja que ha obligado a replantear las agendas de los medios tradicionales. Este trabajo desarrolló el tema: Estudio de los futuros comunicadores y su categoría de prosumidores en el contexto de la globalización.

Este documento tiene como pregunta de investigación: ¿En qué contexto se desenvuelven los futuros comunicadores dentro de la categoría de prosumidores en medio de la globalización? El escenario de cambio y adaptación ha creado una cultura participativa de las audiencias más jóvenes, que no tiene precedentes. Se hace necesario que los medios y agentes implicados asuman su parte de responsabilidad social, sin 
olvidar insistir en la necesidad de una formación crítica de los menores para enfrentarse a los retos mediáticos futuros. Se estableció como objeto de estudio a los Prosumidores.

La metodología empleada para esta investigación fue de tipo cuali-cuantitativa; el estudio fue de campo - descriptivo; se utilizaron como técnicas la encuesta a 70 estudiantes de séptimo y octavo semestre, de la carrera de Comunicación, de la Universidad Nacional de Chimborazo; la entrevista a un docente experto en procesos elearning; y, observación de si el manejo de las herramientas dentro los Complejos de Comunicación Virtual (CCVs) responden a las tendencias actuales. Y, como instrumentos: el cuestionario, la guía de entrevista no estructurada y la ficha de observación.

Los resultados evidenciaron que en la actualidad los prosumidores dentro de la unidad académica están generando nuevos procesos de comunicación que fortalecen la interacción e interrelación con la comunidad virtual.

\section{Desde la perspectiva comunicacional}

No existe discusión sobre la temporalidad del término prosumidor; lo que está claro es que fue planteado en los años setenta por McLuhan \& Nevitt (1972), cuyas dimensiones han ido evolucionando hasta la esfera de las redes sociales, coincidiendo y fortaleciéndose en la convergencia entre los medios tradicionales y los nuevos medios de comunicación. El fenómeno de la convergencia ha sido identificado como una parte esencial de la globalización de la comunicación por el advenimiento, primero, y luego desarrollo de las Tecnologías de la Información y la Comunicación (Tics).

A propósito, Robertson $(1992$, p. 8) en su trabajo sobre la globalización como teoría social y cultura global, observa que el fenómeno "se refiere tanto a la comprensión del mundo como a la intensificación de la conciencia de este como un todo". Es en este marco en el que adquiere todo su relieve el concepto de cultura global, y en el que pueden inscribirse las investigaciones pertenecientes al ámbito anglosajón de los denominados cultural studies, que emplean dicha denominación con una orientación semejante a la que se le asignaba en la antigua distinción naturaleza/ cultura, o con la significación de "software humano general", en palabras de Pieterse (1995).

En este contexto, Zallo en Albornoz (2001) considera que lo global no puede desvincularse de las Tics. Porque ellas están siendo instrumento para la emergencia de una sociedad de la información global. Esta perspectiva, sin dudar, halló el camino para la transformación del capitalismo industrial en capitalismo digital o en capitalismo cognitivo.

Jürgen Habermas (1993, p. 54), reflexiona sobre:

La progresiva racionalización de la sociedad depende de la institucionalización del progreso científico y técnico. En la medida en que la ciencia y la técnica penetran en los ámbitos institucionales de la sociedad, transformando de este modo a las sociedades mismas, empiezan a desmoronarse las viejas legitimaciones. La pérdida que ello implica 
de su capacidad de orientar la acción, y con la tradición cultural en su conjunto, son ora cara de la creciente racionalidad de la acción social.

Es por eso por lo que no debe descartarse la importancia que la mediación tiene en la actualidad; este eje no tan reciente tiene que mirarse como la magnífica oportunidad que los prosumidores tienen para alcanzar el desarrollo de sus propuestas y para encontrar eco en las ciberaudiencias, cada vez más exigentes. A partir de la conceptualización de mediación, vale recordar que, Jesús Martín Barbero fue el primero en hacer uso sistemático del concepto de mediación.

En su trilogía sobre la sociedad red, Castells no tenía, curiosamente, mucho qué decir sobre los medios y la comunicación en particular (...); sin embargo, se centra en la comunicación como clave en la política, economía y todos los campos de la interacción humana de la sociedad red, (Herner \& Tufte, 2013, p. 53).

Para tomar la referencia del profesor español Castells, el poder de la sociedad en red es el poder de la comunicación, no como procesual sino como una categoría que posibilita la interacción e interrelación humana garantía de una plena construcción de sentido de lo social.

"Necesitamos un pensamiento nuevo y diferente en las organizaciones periodísticas para sobrevivir y prosperar en este nuevo panorama de medios. Pero eso no necesariamente significa gente nueva y diferente. Este pensamiento innovador podría venir de la misma gente inteligente y dedicada que ha tenido éxito ejerciendo el periodismo desde antes de que Internet cambiara el juego", (Briggs, 2007, p. 15).

Entonces, desde la propuesta actual el prosumidor mediático produce - consume información, ocupa un papel relevante en el nuevo paradigma de la cultura participativa, propiciado por la interactividad tecnológica (Sandoval \& Aguaded, 2012), que lo caracteriza y que requiere también de una educación emocional (Ferrés \& Piscitelli, 2012). Por lo tanto, un ciudadano - prosumidor será poseedor de una serie de competencias que le permitirán llevar a cabo un conjunto de acciones, como: consumidor de medios y recursos audiovisuales, como productor y creador de mensajes y contenidos críticos, como responsable y creativo. Consumir y producir de manera constructiva y autónoma constituyen los objetivos planteados en lo que se denomina alfabetización mediática, a través de diversas estrategias que favorezcan la generación de emisores críticos. Esta propuesta no es reciente, sino que fue bosquejada desde la comunicación popular de Mario Kaplún (2010) y con los parámetros que entrega la alfabetización audiovisual sobre los usos y hábitos de los niños y jóvenes, en los actuales escenarios digitales (Gabelas, 2010), o formando parte de las redes sociales como ejercicio democrático de los jóvenes prosumidores (García-Galera, 2012; García-Galera \& DelHoyo, 2012).

En contexto, prosumidor es un término acuñado hace un cuarto de siglo por Alvin Toffler; proviene de la fusión de producer y consumer. Un grupo de personas (productores) producían el contenido que el resto (consumidores) compraba en forma de diarios, revistas, programas de radio o TV, etc. Pero hoy, con la aparición de blog y redes sociales, por ejemplo, formas muy sencillas de tener un espacio propio en la red, cualquier 
consumidor de contenidos puede a la vez ser productor de material que otro puede consumir. Esto da lugar a un nuevo tipo de perfil: el prosumidor.

El prosumer describe a millones de participantes en la revolución de la Web 2.0, con cada vez más personas involucradas en la construcción de información cuyo destino es la red y a su vez son consumidores de la misma, creando así un abanico de información en todos los sentidos. El prosumidor que dominó en la sociedad de la primera ola (Toffler, 1980), otra vez se ha constituido en el centro de la acción económica, solo que esta vez lo hace en base a la alta tecnología. Se considera como el resurgimiento del prosumidor, pero de uno de carácter tecnológico. Al término se puede abordar desde diferentes perspectivas: una de las más habituales lo relaciona con el mundo del marketing (Tapscott, Ticoll \& Lowy, 2001; Friedman, 2005; Werner \& Weiss, 2004) y el poder que puede llegar a ejercer en la estructura socioeconómica. Es ésta, sin embargo, una idea que se aleja de la que subyace entre las líneas de este artículo, que lo defiende teniendo en cuenta la visión humanística de aquella persona que, en su afán por consumir, es también capaz de producir. No obstante, lo verdaderamente importante no es tanto saber qué es capaz de producir o consumir, ni reflexionar sobre qué relaciones establece con los medios o con el entorno, sino saber a costa de qué lo hace, con qué valores y con qué principios éticos.

La sociedad actual, enfocada por la globalización y más individualizada que en ningún otro momento, es la que conforman prosumidores de diferente naturaleza como los que enumeran algunos diarios digitales especializados -persuasivos, intermediarios, líderes de opinión, aprendices, escépticos, innovadores, sociales y críticos-. Esta circunstancia, obliga a interrogarse ¿cuál es el perfil de prosumidor ideal? Un individuo productor de nuevos mensajes con capacidad para organizar los recursos necesarios para generar contenidos creativos e innovadores; un revisor de los contenidos que recibe y de los que elabora, desde una mirada crítica, reflexiva y plural; debe ser observador del proceso de producción y emisión del mensaje y su impacto, teniendo en cuenta las posibles audiencias.

El prosumidor debería ser seleccionador de contenidos y recursos adaptados a la era tecnológica y a las nuevas formas de aprendizaje y unificador de criterios de calidad, equidad, inclusión y máxima difusión del mensaje. Otra de las cualidades apunta a convertirse en manipulador de herramientas tecnológicas adaptadas a los nuevos medios de comunicación y a las características de los nuevos mensajes y productos mediáticos, así como un identificador de estereotipos, malas prácticas, abusos y de falta de veracidad de algunos mensajes que se distribuyen a través de los medios de comunicación y las redes sociales, con la posibilidad de deslegitimación de lo publicado. Un prosumidor integral aquel dinamizador de la comunicación y de la interacción entre emisores y receptores, el organizador de los recursos requeridos para la producción de contenidos creativos, críticos y responsables, favoreciendo la participación democrática y el realizador de nuevos mensajes, asumiendo la responsabilidad de cuidar la calidad tecnológica, artística, ética y moral del producto final. 
Es cierto que, la mayoría de ellos encuentran en los medios una herramienta de participar en el entramado social, reconociendo su papel en los procesos de creación y difusión de los valores sociales (Fernández-Beaumont, 2010); quizá sea este el motivo para encontrar artículos que reflexionen sobre la ciudadanía activa. En general, los prosumidores son conscientes también del valor de la información generada y del poder que les confiere. Esta realidad fue identificada hace tiempo por las grandes empresas y por eso recurren a él para analizar tendencias o recabar opiniones con miras a transformar correctamente sus productos. Fernández-Beaumont (2010, p. 15) sugieren que, "el viejo consumidor de medios es pasivo, predecible, aislado y silente; mientras que el nuevo es activo, migratorio, está conectado socialmente, es ruidoso y público".

De acuerdo con Noam Chomsky (1997, p. 60), los medios masivos convencionales promueven la pasividad dirigida sobre sus audiencias: no desean gente con poder de decisión y de participación; buscan una población de consumidores y espectadores políticos pasivos y obedientes; una comunidad tan atomizada y aislada que le resulte imposible reunir sus limitados recursos para convertirse en una fuerza independiente, poderosa que denuncie la concentración de poder. Si el usuario de los medios de comunicación convencionales se ve obligado a soportar la "pasividad dirigida" que denuncia Chomsky, la ecología cultural en la cual se desenvuelve el prosumidor estimula su autonomía. Atento a tal fenómeno, Thomas Friedman, autor del libro La tierra es plana. Breve historia del mundo globalizado del siglo XXI (2005, p. 164) afirma: "Jamás en la historia del planeta tanta gente ha tenido la posibilidad de buscar por sí misma tanta información acerca de tantos temas o acerca de tanta gente".

Finalmente, la colaboración es medular para un comportamiento efectivo de los prosumidores. Los nuevos ambientes comunicativos que desplazan el desarrollo de la web 2.0 -la blogósfera, por ejemplo-, es escenario propicio para el activismo de redes de prosumidores, las cuales han denunciado, por ejemplo, prácticas inescrupulosas de algunas marcas cuyo comportamiento no precisamente corresponde a lo dispuesto en sus códigos de ética.

\section{Metodología}

El presente artículo utiliza el método cuali-cuantitativo; el estudio fue de campo - descriptivo; la técnica escogida fue la encuesta a 70 estudiantes de séptimo y octavo semestre de la Carrera de Comunicación de la Universidad Nacional de Chimborazo; la entrevista a un docente experto en procesos e-learning; y, la observación al manejo de las herramientas dentro los Complejos de Comunicación Virtual (CCVs) responden a las tendencias actuales.

Como instrumentos se aplicaron: el cuestionario, en cuyos ítems se consultó a los alumnos sobre comportamiento de los estudiantes frente a las Tics y a las herramientas de la web 2.0, el manejo de CCVs, el uso de redes sociales y la experiencia como prosumidores; las preguntas a Jorge Cruz, docente universitario y especialista en procesos e-learning se encasillaron en: cuál es la influencia en la educación de las Tics y la 
herramientas de la web 2.0; la construcción de complejos de comunicación virtual como elementos para los prosumidores; diferencias entre herramientas freeware y adware, shareware y software libre; parámetros para la elección de software para la construcción de CCVs; herramientas de análisis para redes sociales y sitios web.

Y la ficha de observación: el comportamiento de los prosumidores respecto a tres parámetros: alimentación de información, evaluación de información y los resultados de los análisis a través de las herramientas de software; el comportamiento de los prosumidores en redes sociales; y, el comportamiento de los prosumidores al obtener habilidades y destrezas de community manager, y la aplicación en sus complejos de comunicación virtual.

Se realizó una necesaria revisión de autores especializados en la temática para construir la fundamentación teórica y fortalecer la discusión de los hallazgos en la aplicación de los instrumentos escogidos.

\section{Hallazgos}

A continuación, se presentan los resultados obtenidos durante los tres momentos de la investigación denominada Estudio de los futuros comunicadores y su categoría de prosumidores en el contexto de la globalización, realizado como parte del Observatorio de Medios y Estudios Sociales:

a) Encuesta a estudiantes de séptimo y octavo semestre, carrera de Comunicación Social de la Unach

Tabla 1. Tics - herramientas Web 2.0 y los prosumidores

\begin{tabular}{|c|c|c|c|c|c|c|c|}
\hline 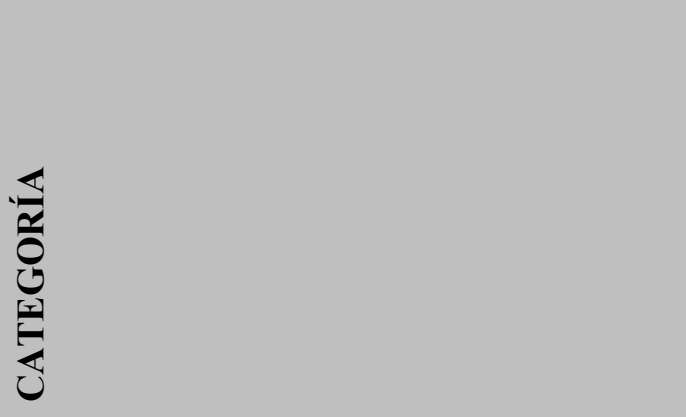 & 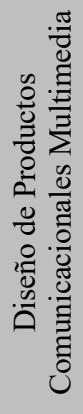 & 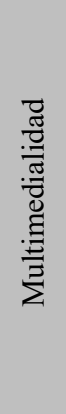 & 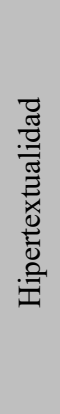 & 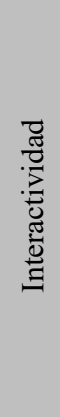 & 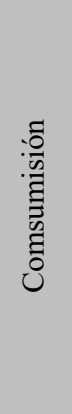 & 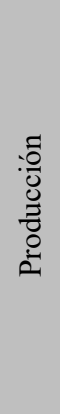 & 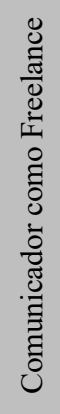 \\
\hline Estudiantes como prosumidores. & & & & & $5 \%$ & $10 \%$ & $85 \%$ \\
\hline Herramientas Web 2.0 & $78 \%$ & & & & $12 \%$ & & \\
\hline Complejos de Comunicación Virtual & & $25 \%$ & $17 \%$ & $22 \%$ & & & \\
\hline Redes Sociales & $15 \%$ & & & $45 \%$ & & & \\
\hline $\begin{array}{l}\text { Comunicación Social - Periodismo y Tics y } \\
\text { Multimedia }\end{array}$ & $35 \%$ & & & & & & $50 \%$ \\
\hline $\begin{array}{l}\text { Herramientas web } 2.0 \text { y Diseño Gráfico asistido } \\
\text { por computador }\end{array}$ & $35 \%$ & & & & $12 \%$ & $18 \%$ & \\
\hline El comunicador apoyado por Software Libre & $40 \%$ & & & & & & $35 \%$ \\
\hline
\end{tabular}

Fuente. Resultados de la investigación

Elaboración. Autores 
Los estudiantes como prosumidores han determinado que dentro de las prioridades se encasillan como el comunicador enfocado a desarrollar habilidades de freelance con un $85 \%$ de incidencia; en el uso de las herramientas de la web 2.0 consideran que las mismas se usan para diseñar productos comunicacionales digitales y multimedia con un 75\%; respecto a los complejos de comunicación virtual toma importancia la Multimedialidad, Hipertextualidad e Interactividad con un 25\% , 17\% y $22 \%$ respectivamente; además, con respecto a la Comunicación Social, Periodismo y Tics y multimedia el 50\% se clasifican comunicador y freelance; las redes sociales dentro de los prosumidores son consideradas como elementos de diseño de productos comunicacionales y herramientas de interactividad con un 45; al referirse al diseño gráfico asistido por computador con las herramientas de la web 2.0 el 35\% considera que son escenciales para el diseño de productos comunicacionales multimedia; el $12 \%$ son parte de la consumisión de información y el 18\% para producción de contenidos e información; finalmente como parte de los prosumidores; el diseño de productos multimedia es realizado en un alto grado por herramientas de software libre en un $40 \%$.

b) Entrevista al Ms. Jorge Cruz, docente universitario y experto en procesos e-Learning

Tabla 2. Prosumidores - Tics y Estudiantes

\begin{tabular}{|c|c|c|c|c|c|}
\hline 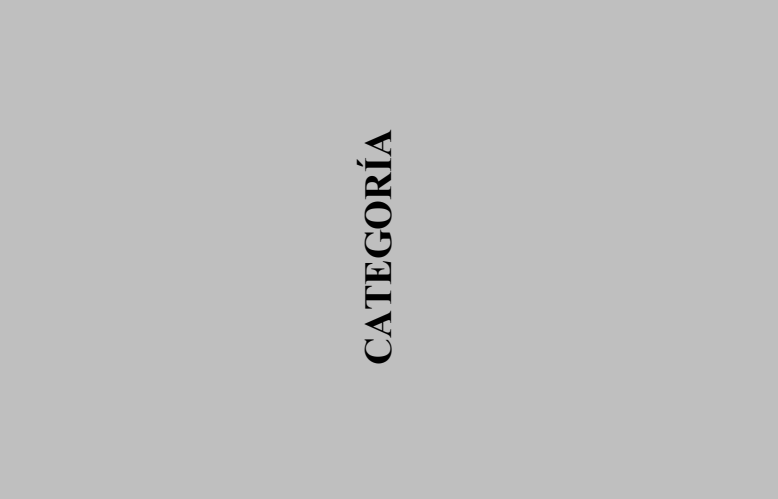 & 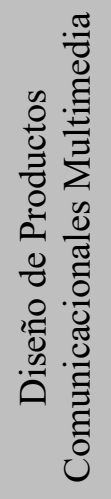 & 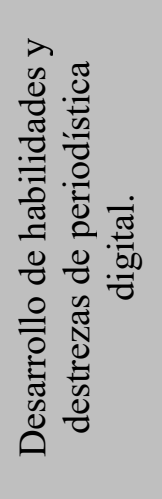 & 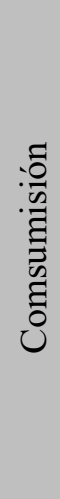 & 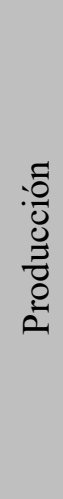 & 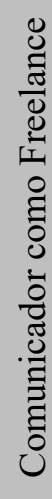 \\
\hline $\begin{array}{l}\text { Influencia en la educación de las Tics y las } \\
\text { herramientas de la web } 2.0\end{array}$ & & $\mathrm{X}$ & $\mathrm{x}$ & & $\mathrm{X}$ \\
\hline $\begin{array}{l}\text { La construcción de complejos de } \\
\text { comunicación virtual como elementos para } \\
\text { los prosumidores }\end{array}$ & $\mathrm{X}$ & & $\mathrm{X}$ & $\mathrm{x}$ & \\
\hline $\begin{array}{l}\text { Diferencias entre herramientas freeware y } \\
\text { adware, shareware y software libre }\end{array}$ & & & & $\mathrm{x}$ & $\mathrm{X}$ \\
\hline $\begin{array}{l}\text { Parámetros para la elección de software para } \\
\text { la construcción de complejos de } \\
\text { comunicación virtuales }\end{array}$ & $\mathrm{X}$ & $\mathrm{X}$ & & $\mathrm{x}$ & $\mathrm{X}$ \\
\hline $\begin{array}{l}\text { Herramientas de análisis para redes sociales } \\
\text { y sitios web }\end{array}$ & & $\mathrm{X}$ & $\mathrm{x}$ & $\mathrm{x}$ & $\mathrm{X}$ \\
\hline
\end{tabular}

Fuente. Resultados de la investigación

Elaboración. Autores 
El experto entrevistado sostiene que es importante la influencia que las Tics y las herramientas de la web 2.0 tienen en la educación de los jóvenes y que en lo posterior influirán en el desarrollo de habilidades y destrezas de Periodismo Digital; respecto de la construcción de Complejos de Comunicación Virtual como elementos para los prosumidores hace referencia a la relevancia que tiene el uso de herramienta adecuada como soporte de la construcción de productos comunicacionales; realiza la diferenciación entre herramientas freeware y adware, shareware y software libre; éstas son usadas en alto grado para convertirse en un comunicador con dotes de freelance; dentro de los parámetros para la elección de software para la construcción de Complejos de Comunicación Virtual, uno de los que más incidencia tuvo es para el Diseño de Productos Comunicacionales Multimedia y como parte del desarrollo de habilidades y destrezas del Periodista Digital. Finamente al evaluar los contenidos e interactividad de las redes sociales y páginas web considera que es necesario desarrollar las habilidades y destrezas del Periodista Digital lo que derivará inevitablemente a convertirlo en un freelance.

\section{Observación}

Tabla 2. Prosumidores - Tics y Estudiantes

\begin{tabular}{|c|c|c|c|c|}
\hline \multirow[t]{2}{*}{ CATEGORÍA } & \multirow[t]{2}{*}{ INDICADORES } & \multicolumn{3}{|c|}{ ESCALA } \\
\hline & & ALTA & MEDIA & BAJA \\
\hline \multirow{3}{*}{$\begin{array}{l}\text { Comportamiento de los } \\
\text { prosumidores en los ejes }\end{array}$} & Alimentación de información & $\mathrm{X}$ & & \\
\hline & Evaluación de la información & $\mathrm{X}$ & & \\
\hline & Herramientas de software & & $\mathrm{X}$ & \\
\hline \multirow{3}{*}{$\begin{array}{l}\begin{array}{l}\text { Comportamiento de los } \\
\text { prosumidores }\end{array} \\
\text { en } \\
\text { herramientas de la web } 2.0\end{array}$} & Redes sociales & & $\mathrm{X}$ & \\
\hline & Complejos de comunicación virtual & $\mathrm{X}$ & & \\
\hline & Páginas web & $\mathrm{X}$ & & \\
\hline \multirow{4}{*}{$\begin{array}{lll}\text { Evaluación } & & \text { del } \\
\text { comportamiento } & \text { de } & \text { los } \\
\text { recursos usados } & \text { por } & \text { los } \\
\text { prosumidores } & & \end{array}$} & Freeware & & $\mathrm{X}$ & \\
\hline & Adware & & $\mathrm{X}$ & \\
\hline & Software libre & $\mathrm{X}$ & & \\
\hline & shareware & & $\mathrm{X}$ & \\
\hline \multirow{4}{*}{$\begin{array}{l}\text { Herramientas de análisis } \\
\text { para redes sociales y sitios } \\
\text { web }\end{array}$} & Análisis de redes sociales & $\mathrm{X}$ & & \\
\hline & $\begin{array}{l}\text { Análisis de } \quad \text { contenidos } \\
\text { multimediales }\end{array}$ & $\mathrm{X}$ & & \\
\hline & Análisis de CCVs & $\mathrm{X}$ & & \\
\hline & $\begin{array}{l}\text { Análisis de accesibilidad } \mathrm{y} \\
\text { usabilidad }\end{array}$ & $\mathrm{X}$ & & \\
\hline
\end{tabular}

Fuente. Resultados de la investigación

Elaboración. Autores 
Para la ficha de observación se utilizó la escala cualitativa, para evaluar varias categorías:

Comportamiento de los prosumidores: quienes tienen una alta influencia son ALIMENTACIÓN DE LA INFORMACIÓN Y EVALUACIÓN DE LA INFORMACIÓN; Comportamiento de los prosumidores en herramientas de la web 2.0; quienes tienen una alta influencia son las Páginas web y los Complejos de Comunicación Virtual; Evaluación del comportamiento de los recursos usados por los prosumidores quien tiene una alta influencia es el software libre que es considerado en la actualidad como una tendencia de uso de programas; al hablar del análisis para redes sociales y sitios web, a los cuatro indicadores analizados, refleja una alta incidencia.

\section{Discusión}

En primera instancia, los resultados alcanzados con la aplicación de la encuesta muestran que el nuevo rol de los estudiantes como prosumidores establece las prioridades como aquel comunicador con competencias para actuar como freelance; las herramientas de la web 2.0 son la base para la elaboración de contenidos atractivos y que respondan a las necesidades de las ciberaudiencias comunicacionales digitales y multimedia con las más altas tasas de incidencia; al hablar de los complejos de comunicación virtual la Multimedialidad, Hipertextualidad e Interactividad son los puntos más importantes; el desarrollo de habilidades y destrezas del Comunicador Freelance complementado con la Comunicación Social, Periodismo, Tics y Multimedia son fundamentales; los prosumidores dentro de las redes sociales se ocupan del diseño y la difusión de productos comunicacionales, complementados con herramientas de interactividad; estos detalles merecen ser tomados en cuenta por su importancia; también, el diseño gráfico asistido por computador es fundamental para la construcción de productos comunicacionales multimedia para el consumo y la producción de información; para concluir, el uso de software libre en el diseño ha tomado importancia por los beneficios que ofrece.

Guillermo López en Rodríguez \& García (2011, pp. 63 - 64) coincide con las particularidades que se reflejan los hallazgos de la prense investigación, una:

Ruptura del tiempo y el espacio, lo que implica instantaneidad (las redes de comunicación basadas en el lenguaje digital son capaces de llevar a cabo la transmisión de datos de una forma mucho más rápida que con los mecanismos convencionales), ruptura de la periodicidad (dichos contenidos pueden actualizarse en cualquier momento y constantemente), universalidad (los contenidos pueden ser consultados desde cualquier otro lugar conectado a la red) y capacidad de almacenamiento. Carácter multimedia, pues se hace acopio de casi todas las formas comunicativas para integrarlas en un mismo soporte como mecanismos de expresión complementarios que dieran lugar, en última instancia, al lenguaje multimedia. Hipertextualidad, porque el internauta cuenta con la posibilidad de convertir el consumo de información en una experiencia comunicativa abierta, es decir, determinar en cuanto receptor activo qué contenidos desea recibir, con qué estructuración, en qué orden, etc. Interactividad, que puede definirse como la capacidad del receptor para tomar decisiones y regular el flujo de la información 
El desarrollo de habilidades y destrezas del Periodismo de Digital son pieza clave en el proceso de aprendizaje de las Tics y de las herramientas de la web 2.0; los prosumidores han contribuido de manera eficiente en la construcción de complejos de comunicación virtual para difundir contenidos multimediales; además de consolidarse como un comunicador con dotes de freelance; los parámetros evaluados para la construcción de complejos de comunicación virtuales, los contenidos multimedia serán los que determinen el proceso de Periodismo Digital complementado con las habilidades $\mathrm{y}$ destrezas de un freelance.

A propósito del presente estudio, es inevitable no referirse a la importante que tiene reconocer que:

Vivimos en una nueva era tecnológica en la que Internet se ha convertido en un medio organizado por usuarios que marcan sus propias reglas a un ritmo acelerado. Es por ello que hablamos de un nuevo concepto de entender la comunicación, la información y el ocio, según una nueva configuración y un nuevo uso social. Los nuevos medios digitales no sólo ha modifican los canales de comunicación, sino también los contenidos, su producción y su consumo (Del Pino, Castelló y Ramos-Soler, 2013, p. 578).

Finalmente, el comportamiento de los prosumidores con las herramientas de la web 2.0 ha sido fundamental en la construcción de los Complejos de Comunicación Virtual; y la influencia del software libre como paradigma de equilibrio y trabajo, para llegar finalmente al proceso de evaluación en el análisis de redes sociales, análisis de contenidos multimediales, análisis de CCVs, análisis de accesibilidad y usabilidad fue de utilidad. La ficha de observación aplicada a los trabajos presentados por los alumnos de la Universidad Nacional de Chimborazo, se presentó como un criterio para identificar un nuevo escenario de lo educativo-comunicacional.

La tecnología se ha convertido en un recurso instruccional fundamental que apoya las dinámicas propias del proceso de enseñanza-aprendizaje, sea éste presencial, a distancia o mixto. El desarrollo tecnológico ha tomado un rumbo antes inimaginable aportando mayores beneficios a la educación. Quienes han apostado por la transformación educativa sustentada en la aproximación constructivista del aprendizaje y la enseñanza, han orientado la praxis pedagógica a flexibilizar los esquemas de enseñanza-aprendizaje; asumir al alumno como responsable último de su propio proceso de aprendizaje, como un sujeto activo que manipula, explora, descubre o inventa; cambiar la mentalidad del profesor, actuando ahora como mediador, guía y facilitador en la construcción de nuevos esquemas de conocimiento por parte del alumno; promover un aprendizaje significativo y permanente, acorde con el contexto en el que se inserta; crear ambientes propicios para el desarrollo de su autonomía moral e intelectual, la motivación y responsabilidad por el estudio y la disposición para cooperar y trabajar colaborativamente, buscando el bien colectivo.

Como un ejercicio de concreción, se propone una clasificación de las herramientas de la web 2.0 que pueden enriquecer la enseñanza de la Comunicación Social y promover los cuatro tipos de aprendizaje que sustentan el modelo de aprendizaje 2.0. En esta clasificación se agrupan las herramientas en torno a dos categorías: herramientas genéricas o transversales (que pueden apoyar todo el proceso de 
aprendizaje) y herramientas específicas (que pueden apoyar el aprendizaje de un área de la disciplina), (Martínez, 2010, p. 189).

\section{Conclusiones}

En este trabajo no se buscó encajar al prosumidor dentro de la definición de periodista próximo a lo ciudadano en alguna de sus facetas, sino en reflexionar sobre las prácticas que desarrollan como productores - mediadores de información y conocimiento a través de los distintos espacios de Internet.

Sin embargo, sí se toma en cuenta esta discusión para seleccionar la metodología mediante la cual se abordó el tema propuesto, se retoman las discusiones sobre el papel que tienen este tipo de espacios como fuentes de información y se rescata la discusión sobre los ideales y prácticas del periodismo como categorías para analizar las prácticas de los prosumers. Sus creaciones son vistas como espacios desde donde los ciudadanos pueden ejercer su derecho de libertad de expresión y de acceso a la información, en miras de una construcción de comunidad, ciudadanía y democracia.

Si bien lo descrito refleja el nuevo panorama del ecosistema mediático ecuatoriano, es importante analizar cómo ha sido el desarrollo de la generación de contenidos en el país y en articular en los alumnos de la Universidad Nacional de Chimborazo. Esta investigación permitió comprender el entorno desde donde surgen espacios ciudadanos de generación de contenidos, y su relación con la producción tradicional de los medios de comunicación.

Tanto en el periodismo como en espacios como blogsfera y los podcasts, la disciplina de verificación depende del hábito o de los esquemas de acción que hayan incorporado los productores. Existe mayor probabilidad de que aquellos prosumers que se inscriben en campos periodísticos reflejen la disciplina de verificación en los espacios que producen para su distribución en la web; sin embargo, será importante en el futuro determinar si los productores de información han adoptado esta práctica, en qué medida se realiza a manera de disciplina para generar información de calidad y cómo las audiencias de estos contenidos exigen también esa verificación.

Los prosumers son individuos que por decisión propia se dedican a producir contenidos con la intención de divulgar la información que han recolectado de sus fuentes y expresar sus puntos de vista. Y esta es la perspectiva en la que se desenvuelven casa adentro, y con sus productos hacia afuera, los estudiantes de la carrera de Comunicación Social de la Universidad Nacional de Chimborazo una vez convertidos en prosumidores. 


\section{Referencias}

Albornoz, L. (2011). Poder, medios, cultura. Paidós. Buenos Aires - Argentina

Briggs, M. (2007). Periodismo 2.0. Knight Fundation. Estados Unidos

Chomsky, N. (1997). Secretos, mentiras y democracia. México: Siglo Veintiuno Editores.

Cornella, A. y Rucabado, S. (2006). El futuro es atreverse hoy. 101 Ideas-Fuerza para entender las próximas décadas. España: Ediciones Deusto.

Del Pino, C.; Castelló, A.; Ramos, I. (2014). Web 2.0 y redes sociales: estudio de las publicaciones científicas en las revistas españolas de comunicación. Revistas Científicas Complutenses - Historia y Comunicación Social. Universidad Complutense de

Madrid. http://revistas.ucm.es/index.php/HICS/article/view/44986/42357. Fecha de consulta: (10-03-2017)

Ferrés, J. \& Piscitelli, A. (2012). Competencia mediática. Propuesta articulada de dimensiones e indicadores. Comunicar 38; 75-82. doi: http://dx.doi.org/10.3916/C38- 2011-02-08. Fecha de consulta: (10-03-2017)

Friedman, T. (2005). La Tierra es plana. Breve historia del mundo globalizado del siglo XXI. España: Mr. Ediciones.

Gabelas, J.A. (2010). Escenarios virtuales, cultura juvenil y educomunicación 2.0. In R. Aparici (Coord.), Educomunicación: más allá del 2.0. (pp. 205-223). Barcelona: Gedisa.

García-Galera, M.C. (2012). Twitéalo: la generación Y y su participación en las redes sociales. Crítica, 985, 34-37.

Habermas, J. (1993). Ciencia y técnica como ideología. México REI

Herner, O. \& Tufte, T. (2013). Comunicación y desarrollo en el mundo mediatizado. Paidós

Kaplún, M. (2010). Una pedagogía de la comunicación. In R. Aparici (Coord.), Educomunicación: más allá del 2.0. (pp. 41-61). Barcelona: Gedisa.

Martínez de Salvo, Fabiola, Herramientas de la Web 2.0 para el aprendizaje 2.0Revista de Artes y Humanidades UNICA [en linea] 2010, 11 (Septiembre-Diciembre): Disponible en: http://www.redalyc.org/articulo.oa?id=170121969008.pdf. Fecha de consulta: (10 de marzo de 2017)

McLuhan M. y Fiore, Q. (1967). The medium is the massage. An inventory of effects. New York: Bantham Books. 
McLuhan, M. (1996). Comprender los medios de comunicación. Las extensiones del ser humano. Barcelona: Paidós Comunicación.

McLuhan, M. y Nevitt, B. (1972). Take Today: the Executive As Dropout. New York: Harcourt Brace Jovanovish.

Pieterse, J. N. (1995). «Globalization as Hybridization». En Featherstone, M., Lash, S., y Robertson, R. (1995). Global Modernities. Londres, Sage, 45-68

Robertson, R. (1992). Globalization: Social Theory and Global Culture. Londres

Rodríguez, A. y García, J. (2011). Aplicación y uso de la web 2.0 y de las redes sociales en la comunicación científica especializada: del marketing viral al usuario activo. Revista Anagramas - Universidad de Medellín. Disponible en: http://www.scielo.org.co/pdf/angr/v9n18/v9n18a05.pdf. Fecha de consulta: (1003-2017)

Sandoval, Y. y Aguaded J.I. (2012). Nuevas audiencias, nuevas responsabilidades. La competencia mediática en la era de la convergencia digital. Icono14 10(2), 8-22, doi: 10.7195/ri14.v10i3.197. Fecha de consulta: (10-03-2017)

Tapscott, D., Tocll, D. y Lowy, A. (2001). Capital digital. El poder de las redes de negocios. España: Taurus Digital.

Toffler, A. (1980). La tercera ola. Barcelona: Plaza \& Janés. UNESCO (2007). Agenda de París o 12 Recomendaciones para la educación en medios. http://goo.gl/BQubIR. Fecha de consulta: 10-03-2017.

Toffler, A. (1981). La tercera ola. México: Edivisión.

Toffler, A. y Toffler, H. (2006). La revolución de la riqueza. España: Deusto.

Werner, K. y Weiss, H. (2006). El libro negro de las marcas. España: De BOLSILLO

\section{Notas}

${ }^{1}$ Julio Bravo Mancero, Doctor (c) en Comunicación e Información Contemporánea de la Universidad Santiago de Compostela; Magíster en Comunicación Corporativa por la Universidad Regional Autónoma de los Andes (2014). Profesor Titular en la Carrera de Comunicación Social de la Universidad Nacional de Chimborazo. Ha publicado los libros: Memorias de la pelota (2006), Reflexiones sobre Comunicación (2009), Palabra Escrita (2010), La rosa de los vientos (2011). Ha sido Director, Editor y Jefe de Redacción de Diario Regional Los Andes, Riobamba. Editor de la Sección B, Diario La Prensa, Riobamba; Director de Relaciones Públicas y Director de Carrera de CS, Unach. https://orcid.org/0000-0002-4468-9247

${ }^{2}$ Carlos Larrea Naranjo, Doctor (c) en Comunicación de la Universidad de La Habana; Magister Business Administration, Universidad Regional Autónoma de los Andes (2012). Profesor Titular en la Carrera de Comunicación Social de la Universidad Nacional de Chimborazo. Ha publicado los libros: Reflexiones sobre Comunicación (2009), En busca de la verdad (2010). Fue Director de Comunicación del Municipio de Riobamba, Municipio de Baños; Director del Departamento de Evaluación Institucional, Unach. 
Consultor Externo de Comunicación; Asesor de Comunicación Política. https://orcid.org/0000-0003-4881$\underline{5666}$

${ }^{3}$ Ramiro Ruales Parreño, Doctor (c) en Comunicación de la Universidad de La Habana; Magíster en Comunicación Corporativa por la Universidad Regional Autónoma de los Andes (2014). Profesor Titular en la Carrera de Comunicación Social de la Universidad Nacional de Chimborazo. Ha publicado los libros: Reflexiones sobre Comunicación (2009). Director de Carrera de CS, Unach; presidente de la Asociación de Profesores, Unach. Experiencia en consultoría y asesoría en Periodismo Digital, Comunicación Audiovisual, Comunicación Política. https://orcid.org/0000-0003-4812-2085 